\title{
WEAK TYPE ESTIMATES \\ FOR A SINGULAR CONVOLUTION OPERATOR ON THE HEISENBERG GROUP
}

\author{
LOUKAS GRAFAKOS
}

\begin{abstract}
On the Heisenberg group $\mathbb{H}^{n}$ with coordinates $(z, t) \in \mathbb{C}^{n} \times \mathbb{R}$, define the distribution $K(z, t)=L(z) \delta(t)$, where $L(z)$ is a homogeneous distribution on $\mathbb{C}^{n}$ of degree $-2 n$, smooth away from the origin and $\delta(t)$ is the Dirac mass in the $t$ variable. We prove that the operator given by convolution with $K$ maps $H^{1}\left(\mathbb{H}^{n}\right)$ to weak $L^{1}\left(\mathbb{H}^{n}\right)$.
\end{abstract}

\section{INTRODUCTION}

$\mathbb{H}^{n}$ is the Lie group with underlying manifold $\mathbb{C}^{n} \times \mathbb{R}$ and multiplication $(z, t) \cdot\left(z^{\prime}, t^{\prime}\right)=\left(z+z^{\prime}, t+t^{\prime}+2 \operatorname{Im} z \cdot \bar{z}^{\prime}\right)$ where, $z \cdot \bar{z}^{\prime}=\sum_{j=1}^{n} z_{j} \bar{z}_{j}^{\prime}$. For $u=(z, t) \in \mathbb{H}^{n}$ we define $|u|=\max _{j}\left(\left|\operatorname{Re} z_{j}\right|,\left|\operatorname{Im} z_{j}\right|,|t|^{1 / 2}\right)$ where, $z=\left(z_{j}\right)$. The norm $|\cdot|$ is homogeneous of degree 1 under the one-parameter group of dilations $D_{r}, r>0$, where $D_{r}(z, t)=\left(r z, r^{2} t\right)$. A Heisenberg group left invariant ball is a set $Q$ of the form

$$
Q=\left\{u \in \mathbb{H}^{n}:\left|u_{0}^{-1} u\right| \leq \delta\right\} \quad \text { for some } u_{0} \in \mathbb{H}^{n}
$$

and some $\delta>0$. The condition $\left|u_{0}^{-1} u\right| \leq \delta$ is equivalent to the pair of conditions $\max _{j}\left(\left|\operatorname{Re} z_{j}-\operatorname{Re} z_{j}^{0}\right|,\left|\operatorname{Im} z_{j}-\operatorname{Im} z_{j}^{0}\right|\right) \leq \delta$ and $\left|t-t_{0}+2 \operatorname{Im} z \cdot \bar{z}_{0}\right| \leq$ $\delta^{2}$, where $u=(z, t), u_{0}=\left(z_{0}, t_{0}\right) . u_{0}$ is the center of the ball $Q$ and $\delta>0$ is the radius. $\mathbb{H}^{n}$ balls are tilted $(2 n+1)$-dimensional rectangles, with maximum tilt from the center of $Q$, proportional to the radius of $Q$ times the Euclidean distance of the projection of the center of $Q$ onto $\mathbb{C}^{n}$, to the origin.

An $\mathbb{H}^{n}$ atom is a function $a(z, t)$ supported in a ball $Q$ and satisfying $\int a(z, t) d z d \bar{z} d t=0$ and $|a| \leq|Q|^{-1} \chi_{Q}$. (We denote by $X_{B}$ the characteristic function of a set $B$.)

Let $L(z)$ be a homogeneous distribution on $\mathbb{C}^{n}$ of degree $-2 n$, which agrees with a smooth function away from the origin. Also let $\delta(t)$ be the Dirac distribution in the $t$ variable. We denote by $K$ the distribution on $\mathbb{H}^{n}$ defined by $K(z, t)=L(z) \delta(t)$. Thus, if $\phi$ is a test function and $\langle K, \phi\rangle$ denotes the action of $K$ on $\phi$, then $\langle K, \phi\rangle=\langle L, \phi(\cdot, 0)\rangle$. Geller and Stein [GS1, GS2]

Received by the editors August 23, 1989 and, in revised form, November 14, 1989.

1980 Mathematics Subject Classification (1985 Revision). Primary 43A80. 
proved that the operator $A: C_{0}^{\infty} \rightarrow C^{\infty}\left(\mathbb{H}^{n}\right)$ given by $A f=f * K$ extends to a bounded operator from $L^{p}$ to $L^{p}$ for $1<p<\infty$. (* denotes the group convolution.) They established their estimates by embedding the operator $A$ in an analytic family of operators $A^{\gamma}$ such that $A^{0}=A$ and by proving an $L^{2}$ and an $L^{p}$ estimate at the endlines of a strip containing 0 . Their result followed by analytic interpolation. It has been an open question whether the operator $A$ is of weak type $(1,1)$. In this paper we establish a weaker estimate, still sharper than the $L^{p}$ boundedness of $A$, namely we prove that $A$ is of weak type $H^{1}$. By this we mean that $A$ extends to an operator that maps $H^{1}\left(\mathbb{H}^{n}\right)$ to weak $L^{1}\left(\mathbb{H}^{n}\right)$. Here $H^{1}\left(\mathbb{H}^{n}\right)$, henceforth $H^{1}$, denotes the Heisenberg group Hardy space, homogeneous under the family of dilations $D r, r>0$, defined as follows: $H^{1}$ is the set of all sums of the form $f=\sum \lambda_{Q} a_{Q}$, where the $Q$ 's are Heisenberg group balls, $\lambda_{Q} \in \mathbb{C}, \sum\left|\lambda_{Q}\right|<+\infty$, and the $a_{Q}$ 's are atoms supported in the corresponding balls $Q$. $\|f\|_{H^{1}}$ is then defined to be the infimum of $\sum\left|\lambda_{Q}\right|$ over all representations of $f$ as $\sum \lambda_{Q} a_{Q}$. Our main result is the following:

Theorem. The operator $A$, as defined before, is of weak type $H^{1}$.

The proof is an application of the method developed in [C]. Some technical difficulties arise because of the geometry of the Heisenberg balls.

\section{Preliminaries For the PROOF}

For $\sigma, \tau \in \mathbb{Z}, \tau \geq \sigma$, we define the class $\widetilde{R}_{\sigma, \tau}$ of all sets of the form

$$
\begin{aligned}
B=\left\{(z, t) \in \mathbb{H}^{n}: \max _{j}\left(\left|\operatorname{Re} z_{j}-\operatorname{Re} z_{j}^{0}\right|,\right.\right. & \left.\left|\operatorname{Im} z_{j}-\operatorname{Im} z_{j}^{0}\right|\right) \leq 2^{\sigma} \\
& \text { and } \left.\left|t-t_{0}+2 \operatorname{Im} z \cdot \bar{z}_{0}\right| \leq 2^{\sigma+\tau}\right\}
\end{aligned}
$$

for all $\left(z_{0}, t_{0}\right) \in \mathbb{H}^{n}$. For $\tau=\sigma, \widetilde{R}_{\sigma, \sigma}$ is the class of all $\mathbb{H}^{n}$ balls of radius $2^{\sigma}$. Given $B \in \widetilde{R}_{\sigma, \tau}$ let $\sigma(B)=\sigma, \tau(B)=\tau$ denote the associated parameters. The corresponding Euclidean rectangle $B_{\text {Eucl }}$ of $B \in \widetilde{R}_{\sigma, \tau}$ is the set

$$
\begin{aligned}
B_{\mathrm{Eucl}}=\left\{(z, t) \in \mathbb{H}^{n}: \max _{j}\left(\left|\operatorname{Re} z_{j}-\operatorname{Re} z_{j}^{0}\right|,\left|\operatorname{Im} z_{j}-\operatorname{Im} z_{j}^{0}\right|\right) \leq 2^{\sigma}\right. \\
\text { and } \left.\left|t-t_{0}\right| \leq 2^{\sigma+\tau}\right\},
\end{aligned}
$$

where $\left(z_{0}, t_{0}\right)$ is the center of $B$. Let $R_{\sigma, \tau}$ denote the class of all sets in $\widetilde{R}_{\sigma, \tau}$ whose corresponding Euclidean rectangles have vertices closest to the origin of the form

$$
\left(i_{1} 2^{\sigma}, \ldots, i_{n} 2^{\sigma}, j_{1} 2^{\sigma}, \ldots, j_{n} 2^{\sigma}, k 2^{\sigma+\tau}\right)
$$

where $i_{1}, \ldots, i_{n}, j_{1}, \ldots, j_{n}, k$ are integers. 
For $B \in \widetilde{R}_{\sigma, \tau}, B^{*}$ will denote an expansion of $B$ by a constant factor that has the following two properties:

(a) If $B^{\prime} \in R_{\sigma^{\prime}, \tau^{\prime}}, \sigma^{\prime} \leq \sigma, \tau^{\prime} \leq \tau$, and $B^{\prime}$ intersects $B$ then $B^{\prime} \subseteq B^{*}$.

(b) If $D \in R_{\sigma^{\prime}, \tau^{\prime}}, \sigma^{\prime} \leq \sigma, \tau^{\prime} \leq \tau$, and the projection of $D$ onto $\mathbb{C}^{n}$ is contained in the projection of $B$ onto $\mathbb{C}^{n}$, then $D^{*} \subseteq B^{*}$.

Note that any larger expansion of $B^{*}$ (for example $B^{* *}, B^{* * *}$ ) also satisfies (a) and (b).

Given $U, V$ subsets of $\mathbb{H}^{n}$, we denote by $U \cdot V$ the set of all $u v$ where $u \in U, v \in V$, and we denote by $U^{-1}$ the set of all $u^{-1}$ where $u \in U$. Following Christ [C], we define the tendril $T(q)$ of a set $q \in \bigcup_{\sigma} \bigcup_{\tau \geq \sigma} R_{\sigma, \tau}$ to be the set $T(q)=q^{*} \cdot\left\{(z, 0):|z| \leq 2^{\tau(q)+1}\right\}$. One can easily check that $|T(q)| \sim 2^{\sigma(q)+(2 n+1) \tau(q)}$. (We denote by $|B|$ the Lebesgue measure of the set $B$.)

For the proof of our theorem, we may assume that a given $f \in H^{1}$ is a finite sum $\sum \lambda_{Q} a_{Q}$, where $\sum\left|\lambda_{Q}\right| \leq 2\|f\|_{H^{1}}$. Once the theorem is proved for such $f$ the general case will follow by a limiting argument. We may also assume that each $\lambda_{Q}$ in the decomposition of $f$ is positive since we can always multiply an atom by a scalar of modulus one to achieve this. Finally, we can assume that for any ball $Q$ in the decomposition of $f$ we have $\sigma(Q) \in \mathbb{Z}$. The general case follows from the observation that every ball $Q$ is contained in a ball $Q^{\prime}$ with comparable measure and with $\sigma\left(Q^{\prime}\right) \in \mathbb{Z}$. Let us call $\mathscr{F}$ the collection of balls appearing in the atomic decomposition of $f \in H^{1}$.

We are now given an $\alpha>0$ and a finite collection $\mathscr{F}$ of balls $Q$ with $\sigma(Q) \in \mathbb{Z}$ and with associated scalars $\lambda_{Q}>0$. The first of the following two lemmas is valid in any space of homogeneous type $X$. For our purposes $X=\mathbb{H}^{n}$.

Lemma 1. Given $\alpha>0$ and $\mathscr{F}$ as above, there exists a collection of balls $\{S\}$ such that:

The balls obtained by shrinking the $S$ 's by a fixed constant factor are pairwise disjoint and no point in $X$ is contained in more than $M S$ 's. $M$ is a constant depending only on $X$.

$$
\begin{gathered}
\sum_{S}|S| \leq C \alpha^{-1} \sum_{Q \in \mathscr{F}} \lambda_{Q} . \\
\sum_{Q \subset S^{*}} \lambda_{Q} \leq C \alpha|S| . \\
\left\|\sum_{Q \nsubseteq \text { any } S^{*}} \lambda_{Q}|Q|^{-1} \chi_{Q}\right\|_{L^{\infty}} \leq C \alpha .
\end{gathered}
$$


Proof. Let $F=\sum_{Q \in \mathscr{F}} \lambda_{Q}|Q|^{-1} \chi_{Q^{*}}$ and let

$$
\Omega=\left\{x \in X: \sup _{\substack{B \ni x \\ B \text { balls }}}|B|^{-1} \int_{B} F>\alpha\right\} .
$$

$\Omega$ is an open set. Then by [CW, Theorem (1.3), p. 70], there exists a sequence of balls $S_{j}$ such that $\Omega=\bigcup_{j} S_{j}$, a fixed dilate of every $S_{j}$ meets the complement of $\Omega$, and such that (1.1) is satisfied. (The first statement in (1.1) is not explicitly mentioned in the statement of Theorem (1.3) in [CW] but follows easily from the proof.) Using the fact that the Hardy-Littlewood maximal function in a space of homogeneous type is of weak type $(1,1)$ [CW, Theorem (2.1), p. 71] and with the help of (1.1) we immediately derive (1.2). We only need to prove (1.3) and (1.4). For fixed $j$ we have

$$
\sum_{Q \subseteq S_{j}^{*}} \lambda_{Q}=\int \sum_{Q \subseteq S_{j}^{*}} \lambda_{Q}|Q|^{-1} \chi_{Q} \leq C\left|S_{j}\right|\left|S_{j}^{*}\right|^{-1} \int_{S_{j}^{*}} F \leq C \alpha\left|S_{j}\right|
$$

The last inequality is valid because a fixed dilate of $S_{j}$ meets the complement of $\Omega$. To check (1.4) fix $x_{0} \in X$. Let $Q_{0}$ be a ball with the smallest possible radius that contains $x_{0}$ and is not contained in any $S_{j}$. Then

$$
\begin{aligned}
& \sum_{Q \nsubseteq \text { any } S_{j}^{*}} \lambda_{Q}|Q|^{-1} \chi_{Q}\left(x_{0}\right) \leq \sum_{Q \nsubseteq \text { any } S_{j}^{*}} \lambda_{Q}|Q|^{-1} \chi_{Q^{*}}\left(x_{0}\right) \\
& =\left|Q_{0}\right|^{-1} \int_{Q_{0}} \sum_{Q \nsubseteq \text { any } S_{j}^{*}} \lambda_{Q}|Q|^{-1} \chi_{Q^{*}} \leq\left|Q_{0}\right|^{-1} \int_{Q_{0}} F .
\end{aligned}
$$

Let $S_{0}$ be any $S$ containing $x_{0}$. If $Q_{0}$ had smaller radius than $S_{0}$ then $Q_{0} \subseteq S_{0}^{*}$, which is impossible. Thus $S_{0}$ has no larger radius than $Q_{0}$ which implies that $S_{0} \subseteq Q_{0}^{*}$. Therefore some dilate of $Q_{0}^{*}$, call it $Q_{0}^{\#}$, meets the complement of $\Omega$. We then have $\left|Q_{0}^{\#}\right|^{-1} \int_{Q_{0}^{*}} F \leq \alpha$. Finally $\left|Q_{0}\right|^{-1} \int_{Q_{0}} F$ $\leq C\left|Q_{0}^{\#}\right|^{-1} \int_{Q_{0}^{*}} F \leq C \alpha$ and thus (1.4) holds. This concludes the proof of Lemma 1.

Call $\mathscr{C}$ the subcollection of $\mathscr{F}$ consisting of all balls $Q$ contained in $S^{*}$ for some $S$ as in Lemma 1.

Lemma 2. Given an $\alpha>0$, and $\mathscr{F}$ and $\mathscr{C}$ as above we can find a measurable set $E \subseteq \mathbb{H}^{n}$ and a function $\kappa: \mathscr{C} \rightarrow \mathbb{Z}$ such that

$$
|E| \leq C \alpha^{-1} \sum_{Q \in \mathscr{F}} \lambda_{Q}
$$

$$
\begin{gathered}
Q \cdot\left\{(z, 0):|z| \leq 2^{j+1}\right\} \subseteq E \text { for all } Q \in \mathscr{C} \text { and all } j<\kappa(Q), \\
\text { if } Q \subset S^{*} \text { then } \kappa(Q)>\sigma\left(S^{*}\right) \geq \sigma(Q),
\end{gathered}
$$




$$
\sum_{\substack{Q \subset q^{*} \\ \kappa(Q) \leq \tau}} \lambda_{Q} \leq 2^{2 n+1} \alpha 2^{\sigma+(2 n+1) \tau}
$$

for any $\sigma, \tau \in \mathbb{Z}, \tau \geq \sigma$, and any $q \in R_{\sigma, \tau}$.

(Here $B^{*}$ denotes any expansion of $B \in \bigcup_{\sigma} \bigcup_{\tau \geq \sigma} \widetilde{R}_{\sigma, \tau}$ that satisfies (a) and (b).) The constant $C$ depends on the dimension $n$ and not on $\alpha, \mathscr{F},\left\{\lambda_{Q}\right\}$.

Proof. Find a $\tau_{0}>\sigma(Q)$ for all $Q \in \mathscr{C}$ such that $\sum_{Q \in \mathscr{C}} \lambda_{Q}<\alpha 2^{2(n+2) \tau_{0}}$. Initially set $\mathscr{C}_{1}=\varnothing$ and $\mathscr{C}_{2}=\varnothing$. Also set $\tau_{1}=\tau_{0}-1, \sigma_{1}=\tau_{1}$. Select all $q \in R_{\sigma_{1}, \tau_{1}}$ such that

$$
\sum_{\substack{Q \subset q^{*} \\ Q \in \mathscr{\mathscr { E }} \backslash\left(\mathscr{E}_{1} \cup \mathscr{C}_{2}\right)}} \lambda_{Q}>\alpha 2^{\sigma_{1}+(2 n+1) \tau_{1}} .
$$

Any $Q$ contained in $q^{*}$ for some $q$ selected is assigned to $q$ and is placed in $\mathscr{C}_{1}$. Notice that any $Q$ of class $\mathscr{C}_{1}$ is assigned to at most $K$ many $q$ selected, where $K$ is a constant depending only on the definition of $q^{*}$. This was step $\left(\sigma_{1}, \tau_{1}\right)$ of the induction. Now set $\sigma_{2}=\sigma_{1}-1$. Select all $q \in R_{\sigma_{2}, \tau_{1}}$ such that

$$
\sum_{\substack{Q \subset q^{*} \\ Q \in \mathscr{E} \backslash\left(\mathscr{E}_{1} \cup \mathscr{C}_{2}\right)}} \lambda_{Q}>\alpha 2^{\sigma_{2}+(2 n+1) \tau_{1}} .
$$

Any $Q$ contained in $q^{*}$ for some $q$ selected is assigned to $q$ and is placed in $\mathscr{C}_{1}$. This was step $\left(\sigma_{2}, \tau_{1}\right)$ of the induction. Continue similarly until we reach a $\sigma$ smaller than $\min _{Q \in \mathscr{C}} \sigma(Q)$. Then place in $\mathscr{C}_{2}$ all $Q$ with $\sigma(Q)=\tau_{1}$ which are not already in $\mathscr{C}_{1}$.

Now set $\tau_{2}=\tau_{1}-1, \sigma_{2}=\tau_{2}$. Select all $q \in R_{\sigma_{2}, \tau_{2}}$ such that

$$
\sum_{\substack{Q \subset q^{*} \\ Q \in \mathscr{E} \backslash\left(\mathscr{E}_{1} \cup \mathscr{C}_{2}\right)}} \lambda_{Q}>\alpha 2^{\sigma_{2}+(2 n+1) \tau_{2}} .
$$

Any $Q$ contained in $q^{*}$ for some $q$ selected is assigned to $q$ and is placed in $\mathscr{C}_{1}$. We just described step $\left(\sigma_{2}, \tau_{2}\right)$ of the induction. Repeat this procedure until all the $\sigma$ 's are exhausted and then place in $\mathscr{C}_{2}$ all $Q \in \mathscr{C}$ with $\sigma(Q)=\tau_{2}$ which are not already in $\mathscr{C}_{1}$. Continue the double induction until we reach a $\tau<\min _{Q \in \mathscr{C}} \sigma(Q)$.

We have now split $\mathscr{C}$ into two disjoint classes $\mathscr{C}_{1}$ and $\mathscr{C}_{2}$. For $Q \in \mathscr{C}_{1}$ define $\kappa(Q)=\max _{q, S}\left(1+\tau(q), 1+\sigma\left(S^{*}\right)\right)$, where the maximum is taken over all $q$ such that $Q$ is assigned to $q$ and over all $S$ such that $Q \subset S^{*}$. For $Q \in \mathscr{C}_{2}$ define $\kappa(Q)=\max _{S}\left(1+\sigma\left(S^{*}\right)\right)$, where the maximum is taken over all $S$ such that $Q \subset S^{*}$. (2.3) is now clearly satisfied. To simplify our notation, for $q \in \bigcup_{\sigma} \bigcup_{\tau \geq \sigma} R_{\sigma, \tau}$ set $\Lambda(q)=\sum_{Q \subset q^{*}} \lambda_{Q}$, where the sum is taken over all 
$Q \in \mathscr{C}$ not yet placed in $\mathscr{C}_{1} \cup \mathscr{C}_{2}$ at step $(\sigma(q), \tau(q))$. To prove (2.4) fix $q \in R_{\sigma, \tau}$ and consider two cases. If $q$ was not selected then

$$
\alpha 2^{\sigma+(2 n+1) \tau} \geq \Lambda(q) \geq \sum_{\substack{Q \subset q^{*} \\ \kappa(Q) \leq \tau}} \lambda_{Q} .
$$

Suppose now that $q$ was selected at the step $(\sigma, \tau)$ of the induction. Let us assume that (2.4) fails, i.e.,

$$
\sum_{\substack{Q \subset q^{*} \\ \kappa(Q) \leq \tau}} \lambda_{Q}>2^{2 n+1} \alpha 2^{\sigma+(2 n+1) \tau} .
$$

Note that for $\left(q^{\prime}\right)^{*} \supset q^{*}$

$$
\Lambda\left(q^{\prime}\right) \geq \Lambda(q) \geq \sum_{\substack{Q \subset q^{*} \\ \kappa(Q) \leq \tau}} \lambda_{Q} .
$$

It follows that, at least when $\tau<\tau_{1}$, the unique $q^{\prime} \in R_{\sigma, \tau+1}$ which contains $q$ would have been selected at step $(\sigma, \tau+1)$; a contradiction because all $Q \in \mathscr{C}$ contained in $q^{*}$ would be contained in $\left(q^{\prime}\right)^{*}$ and therefore they would have been placed in $\mathscr{C}_{1}$ at a previous step. When $\tau=\tau_{1}$ choose $q^{\prime} \in R_{\sigma+1, \tau}$ such that $q_{\text {Eucl }}^{\prime} \supset q_{\text {Eucl }}$. Then by (b), $q^{*} \subset\left(q^{\prime}\right)^{*}$ and the previous argument leads to a contradiction. Define $E=\bigcup_{q \text { selected }} T(q) \cup \bigcup_{S} S^{+}$, where for any $S$, $S^{+}=S^{*} \cdot\left\{(z, 0):|z| \leq 2^{\sigma\left(S^{*}\right)+2}\right\}$. Since

$$
\left|\bigcup_{S} S^{+}\right| \leq \sum_{S}\left|S^{+}\right| \leq C \sum_{S}|S| \leq C \alpha^{-1} \sum_{Q \in \mathscr{F}} \lambda_{Q},
$$

to prove (2.1) it will suffice to bound $\left|\bigcup_{q \text { selected }} T(q)\right|$. We have

$$
\begin{aligned}
& \sum_{q \text { selected }}|T(q)| \leq C \sum_{q \text { selected }} 2^{\sigma(q)+(2 n+1) \tau(q)} \\
& \leq C \alpha^{-1} \sum_{q \text { selected }} \bigwedge(q) \leq C \alpha^{-1} \sum_{q \text { selected } Q} \sum_{\text {assigned to } q} \lambda_{Q} \\
& \leq C \alpha^{-1} \sum_{Q \in \mathscr{E}_{1}} \lambda_{Q} \#\{q: Q \text { is assigned to } q\} \\
& \leq K C \alpha^{-1} \sum_{Q \in \mathscr{E}_{1}} \lambda_{Q} \leq C \alpha^{-1} \sum_{Q \in \mathscr{F}} \lambda_{Q} .
\end{aligned}
$$

Finally, we check (2.2). If $Q \in \mathscr{C}_{1}$ and $j<\kappa(Q)$ then

$$
Q \cdot\left\{(z, 0):|z| \leq 2^{j+1}\right\} \subseteq q \cdot\left\{(z, 0):|z| \leq 2^{\tau(q)+1}\right\}=T(q) \subseteq E,
$$

where $Q$ is assigned to $q$. If $Q \in \mathscr{C}_{2}$, let $S_{0}$ have the largest radius among all $S$ such that $Q \subset S^{*}$. Then $\kappa(Q)=1+\sigma\left(S_{0}^{*}\right)$ and for $j<\kappa(Q)$ 


$$
Q \cdot\left\{(z, 0):|z| \leq 2^{j+1}\right\} \subseteq S_{0}^{+} \subseteq E .
$$

The lemma is now proved.

\section{Proof of THE THEOREM}

We must show that

$$
\left|\left\{u \in \mathbb{H}^{n}|(A f)(u)|>\alpha\right\}\right| \leq \frac{C}{\alpha}\|f\|_{H^{1}} .
$$

We split $f$ as $g+b$, where

$$
b=\sum_{Q \in \mathscr{B}} \lambda_{Q} a_{Q}, \quad g=\sum_{Q \in \mathscr{F} \backslash \mathscr{B}} \lambda_{Q} a_{Q} .
$$

By (1.4), $\|g\|_{L^{\infty}} \leq C \alpha$ and thus

$$
\|g\|_{L^{2}}^{2} \leq C \alpha\|g\|_{L^{1}} \leq C \alpha \sum_{Q \in \mathscr{F} \backslash \mathscr{Q}} \lambda_{Q} \leq \frac{C}{\alpha} \sum_{Q \in \mathscr{F}} \lambda_{Q} \leq \frac{C}{\alpha}\|f\|_{H^{1}} .
$$

The $L^{2}$ boundedness of $A$ in [GS2] gives

$$
\left|\left\{u \in \mathbb{H}^{n}:|(A g)(u)|>\frac{\alpha}{2}\right\}\right| \leq \frac{4}{\alpha^{2}}\|A g\|_{L^{2}}^{2} \leq \frac{C}{\alpha^{2}}\|g\|_{L^{2}}^{2} \leq \frac{C}{\alpha}\|f\|_{H^{1}} .
$$

Therefore (1) will follow from

$$
\left|\left\{u \in \mathbb{H}^{n}:|(A b)(u)|>\frac{\alpha}{2}\right\}\right| \leq \frac{C}{\alpha}\|f\|_{H^{1}} .
$$

Because of (2.1) and of the assumption $\sum_{Q \in \mathscr{F}} \lambda_{Q} \leq 2\|f\|_{H^{1}}$, (2) will be a corollary of

$$
\left|\left\{u \in \mathbb{H}^{n} \backslash E:|(A b)(u)|>\frac{\alpha}{2}\right\}\right| \leq \frac{C}{\alpha} \sum_{Q \in \mathscr{E}} \lambda_{Q}
$$

which in turn will follow from

$$
\|A b\|_{L^{2}\left(H^{n} \backslash E\right)}^{2} \leq C \alpha \sum_{Q \in \mathscr{C}} \lambda_{Q}
$$

with the aid of Chebychev's inequality.

The rest of the paper is devoted to the proof of (3).

Fix $\phi \in C_{0}^{\infty}\left(\mathbb{C}^{n}\right)$ supported in $1 / 2 \leq|z| \leq 2$ such that

$$
\sum_{j \in \mathbb{Z}} \phi\left(2^{-j} z\right)=1 \text { for all } z \in \mathbb{C}^{n}-\{0\} .
$$

We decompose the operator $A=\sum_{j} A_{j}$, where $A_{j}$ is given by convolution with $K_{j}(z, t)=L(z) \phi\left(2^{-j} z\right) \delta(t)$. Since all balls $Q$ appearing in the definition of $b$ are in $\mathscr{C}$, in the remainder of the paper every $Q$ considered is in $\mathscr{C}$. This restriction is assumed to hold in all sums below. 
To treat (3) fix $u \in \mathbb{H}^{n} \backslash E$ and write

$$
\begin{aligned}
A b(u) & =A\left(\sum \lambda_{Q} a_{Q}\right)(u) \\
& =\sum_{j}\left(\left(\sum \lambda_{Q} a_{Q}\right) * K_{j}\right)(u) \sum_{Q}\left(\lambda_{Q} a_{Q} * \sum_{j \geq \kappa(Q)} K_{j}\right)(u) \\
& =\sum_{s \geq 0} \sum_{j \in \mathbb{Z}}\left[\sum_{\kappa(Q)=j-s} \lambda_{Q} a_{Q} * K_{j}\right](u) .
\end{aligned}
$$

If we set

$$
B_{k}=\sum_{\kappa(Q)=k} \lambda_{Q} a_{Q}, \quad k \in \mathbb{Z},
$$

(3) will be a consequence of

$$
\left\|\sum_{j \in \mathbb{Z}}\left(B_{j-s} * K_{j}\right)\right\|_{L^{2}\left(\mathbb{H}^{n}\right)}^{2} \leq C \alpha 2^{-s} \sum_{\kappa(Q)=j-s} \lambda_{Q} \text { for all } s \geq 0 .
$$

Without loss of generality, we may assume that $K(z, t)=L(z) \delta(t)$ is a realvalued distribution. Expanding the square out, we write the left-hand side of (4) as:

$$
\text { (5) } \begin{aligned}
\sum_{j \in \mathbb{Z}}\left[\left\|B_{j-s} * K_{j}\right\|_{L^{2}}^{2}+2\right. & \sum_{j-3<k<j} \int\left(B_{j-s} * K_{j}\right)\left(B_{k-s} * K_{k}\right) d z d \bar{z} d t \\
& \left.+2 \sum_{k \leq j-3} \int\left(B_{j-s} * K_{j}\right)\left(B_{k-s} * K_{k}\right) d z d \bar{z} d t\right] .
\end{aligned}
$$

We will treat the first and the third term inside the brackets in (5). The estimate for the second term in (5) will follow from the estimate for the first term with the use of the Cauchy-Schwarz inequality. We therefore only need to prove

$$
\begin{aligned}
& \sum_{j \in \mathbb{Z}}\left[\left\|B_{j-s} * K_{j}\right\|_{L^{2}}^{2}+\sum_{k \leq j-3} \int\left(B_{j-s} * K_{j}\right)\left(B_{k-s} * K_{k}\right) d z d \bar{z} d t\right] \\
& \leq C \alpha 2^{-s} \sum_{\kappa(Q)=j-s} \lambda_{Q} .
\end{aligned}
$$

By rescaling, it suffices to prove (6) when $j=0$. Our proof will be complete if we can show:

$$
\begin{gathered}
\left\|B_{-s} * K_{0}\right\|_{L^{2}}^{2} \leq C \alpha 2^{-s} \sum_{\kappa(Q)=-s} \lambda_{Q} \text { and } \\
\sum_{k \leq-3}\left|\int\left(B_{-s} * K_{0}\right)\left(B_{k-s} * K_{k}\right) d z d \bar{z} d t\right| \leq C \alpha 2^{-s} \sum_{\kappa(Q)=-s} \lambda_{Q} .
\end{gathered}
$$


We start with the proof of (7). We will need two lemmas.

Lemma 3. For any ball $Q$ with $\sigma(Q)<0,\left\|a_{Q} * K_{0}\right\|_{L^{\infty}} \leq C 2^{-\sigma(Q)}$. Proof. Fix a $Q$ with $\sigma(Q)=\sigma<0$. We have

$$
\begin{aligned}
\left|\left(a_{Q} * K_{0}\right)(z, t)\right| & =\left|\int_{\mathbb{C}^{n}} a_{Q}(z-w, t-2 \operatorname{Im} z \cdot \bar{w}) L_{0}(w) d w d \bar{w}\right| \\
& \leq C|Q|^{-1}\left|S_{Q}(z, t)\right|=C 2^{-(2 n+2) \sigma}\left|S_{Q}(z, t)\right|,
\end{aligned}
$$

where

$$
S_{Q}(z, t)=\left\{w \in \mathbb{C}^{n}: \frac{1}{2} \leq|w| \leq 2 \text { such that }(z-w, t-2 \operatorname{Im} z \cdot \bar{w}) \in Q\right\} .
$$

Clearly

$$
S_{Q}(z, t)=\left\{w \in \mathbb{C}^{n}: \frac{1}{2} \leq|w| \leq 2 \text { such that }(w, 0) \in Q^{-1}(z, t)\right\} .
$$

(We set $Q^{-1}(z, t)=Q^{-1} \cdot\{(z, t)\}$.) Since $Q$ is a left-invariant ball, $Q_{0}=$ $Q^{-1}(z, t)$ is a right-invariant ball of radius $2^{\sigma}$, centered say at $\left(z_{1}, t_{1}\right)$. The set $S_{Q}(z, t)$ is empty unless $\left|z_{1}\right| \sim 1$. This observation implies that the maximum tilt of $Q_{0}$ from its center is $\leq C 2^{\sigma}$. Thus $S_{Q}(z, t)$ is contained in

$$
\left\{w \in \mathbb{C}^{n}:\left|w-z_{1}\right| \leq 2^{\sigma},\left|2 \operatorname{Im} z_{1} \cdot \bar{w}\right| \leq C 2^{2 \sigma}\right\} .
$$

Setting $\zeta=w-z_{1}$, to prove the lemma it will suffice to show that

$$
\mid\left\{\zeta \in \mathbb{C}^{n}:|\zeta| \leq 2^{\sigma} \text { and }\left|\operatorname{Im}\left(\zeta \cdot \bar{z}_{1}\right)\right| \leq C 2^{2 \sigma}\right\} \mid \leq C 2^{(2 n+3) \sigma} .
$$

The latter becomes obvious when we introduce a rotation that takes $z_{1}$ to the point $(a+i 0,0+i 0, \ldots, 0+i 0) \in \mathbb{C}^{n}$, where $|a| \leq C$.

Given $f(z, t)$, a function on the Heisenberg group, let $\tilde{f}$ denote the function $\tilde{f}(z, t)=f\left((z, t)^{-1}\right)=f(-z,-t)$. Note that for all $f, g, h$, functions on any group with Haar measure $d x$, the following is valid:

$$
\int(f * g) h d x=\int f(h * \tilde{g}) d x .
$$

To prove (7) we will need one more lemma.

Lemma 4. $K_{0} * \tilde{K}_{0}$ has compact support and satisfies

$$
\left|\left(K_{0} * \tilde{K}_{0}\right)(z, t)\right| \leq C(|z|+|t|)^{-1}, \quad\left|\nabla\left(K_{0} * \tilde{K}_{0}\right)(z, t)\right| \leq C(|z|+|t|)^{-2} .
$$

Proof. Let $\psi$ be a test function. Then

$$
\begin{aligned}
\left\langle K_{0} * \tilde{K}_{0}, \psi\right\rangle & =\left\langle K_{0}, \psi * K_{0}\right\rangle=\int_{\mathbb{C}^{n}} L_{0}(w)\left(\psi * K_{0}\right)(w, 0) d w d \bar{w} \\
& =\int_{\mathbb{C}^{n}} L_{0}(w) \int_{\mathbb{C}^{n}} \psi(w-\zeta,-2 \operatorname{Im} w \cdot \bar{\zeta}) L_{0}(\zeta) d \zeta d \bar{\zeta} d w d \bar{w} \\
& =\int_{\mathbb{C}^{n}} \int_{\mathbb{C}^{n}} L_{0}(z+\zeta) \psi(z,-2 \operatorname{Im} z \cdot \bar{\zeta}) L_{0}(\zeta) d \zeta d \bar{\zeta} d z d \bar{z}
\end{aligned}
$$


Let $\zeta=\left(\zeta_{1}, \zeta_{2}, \ldots, \zeta_{n}\right), \quad \zeta_{j}=x_{j}+i y_{j}, 1 \leq j \leq n$. Fix $l: 1 \leq l \leq n$ and denote by $z^{\prime}, \zeta^{\prime} \in \mathbb{C}^{n-1}$ the points $z, \zeta$ whose $l$ th variable is deleted. Change variables in (9) by setting

$$
t=2 \operatorname{Im} z \cdot \bar{\zeta}=-2 \operatorname{Im} z_{l} \bar{\zeta}_{l}-2 \operatorname{Im} z^{\prime} \cdot \bar{\zeta}^{\prime}
$$

and

$$
s=-2 \operatorname{Re} z \cdot \bar{\zeta}=-2 \operatorname{Re} z_{l} \bar{\zeta}_{l}-2 \operatorname{Re} z^{\prime} \cdot \bar{\zeta}^{\prime} .
$$

Then $\zeta_{k}=\left(s-i t+2 \bar{z}^{\prime} \cdot \zeta^{\prime}\right) /-2 \bar{z}_{l}$ and $\left|\operatorname{det}\left(\partial\left(x_{l}, y_{l}\right) / \partial(s, t)\right)\right|=\frac{1}{4}\left|z_{l}\right|^{-2}$.

We can rewrite (9) as

$$
\text { (10) } \int_{\mathbb{C}^{n} \times \mathbb{R}} \psi(z, t)\left[\frac{1}{4}\left|z_{l}\right|^{-2} \int_{\mathbb{C}^{n-1}} \int_{\mathbb{R}} L_{0}(z+\tilde{\zeta}) L_{0}(\tilde{\zeta}) d s d \zeta^{\prime} d \bar{\zeta}^{\prime}\right] d z d \bar{z} d t
$$

where

$$
\tilde{\zeta}=\left(\zeta_{1}, \ldots, \zeta_{l-1}, \frac{s-i t+2 \bar{z}^{\prime} \cdot \zeta^{\prime}}{-2 \bar{z}_{l}}, \zeta_{l+1}, \ldots, \zeta_{n}\right) .
$$

It follows that $\left(K_{0} * \tilde{K}_{0}\right)(z, t)$ is equal to the expression inside the brackets in (10). Since $L_{0}$ is supported in $|z| \leq 2$ we must have

$$
\left|z^{\prime}+\zeta^{\prime}\right|+\left|z_{l}+\frac{s-i t+2 \bar{z}^{\prime} \cdot \zeta^{\prime}}{-2 \bar{z}_{l}}\right| \leq C
$$

which implies $\left.|-2| z_{l}\right|^{2}+s+2 \operatorname{Re} \bar{z}^{\prime} \cdot \zeta^{\prime}|\leq C| z_{l} \mid$. Therefore, once $\zeta^{\prime}$ is fixed, $s$ varies over a set of measure $\leq C\left|z_{l}\right|$. It follows that

$$
\left|\left(K_{0} * \tilde{K}_{0}\right)(z, t)\right| \leq \frac{1}{4}\left|z_{l}\right|^{-2} \int_{\left|\zeta^{\prime}\right| \leq C} \int_{s}\left\|L_{0}\right\|_{L^{\infty}}^{2} d s d \zeta^{\prime} \leq C\left|z_{l}\right|^{-1} .
$$

Since $l$ was arbitrary in $\{1,2, \ldots, n\}$ we get $\left|\left(K_{0} * \tilde{K}_{0}\right)(z, t)\right| \leq C|z|^{-1}$. It also follows from (11) that $\left|t-2 \operatorname{Im} \bar{z}^{\prime} \cdot \zeta^{\prime}\right| \leq C\left|z_{k}\right|$ which implies that

$$
|t| \leq C\left|z_{k}\right|+2\left|z^{\prime}\right|\left|\zeta^{\prime}\right| \leq C\left(\left|z_{k}\right|+\left|z^{\prime}\right|\right) \leq C|z| \text {. }
$$

Thus on the support of $\left(K_{0} * \tilde{K}_{0}\right)(z, t),|t| \leq c|z|$ and the asserted bound follows. The estimate for the gradient can be proved similarly.

We now begin the proof of (7).

First fix $s \geq 0$ and assign each $Q$ occurring in (7) to some (but only one) element $q \in R_{\sigma(Q), 0}$ such that $Q$ intersects $q$ (hence $Q \subset q^{*}$ ). For $q$ in $R_{\sigma, 0}$ $(\sigma<0)$ write

$$
A_{q}=\sum_{Q \text { assigned to } q} \lambda_{Q} a_{Q}, \quad \lambda_{q}=\sum_{Q \text { assigned to } q} \lambda_{Q} .
$$

Also split every $q \in R_{\sigma, 0}(\sigma<-s)$ in $2^{s}, q_{j} \in R_{\sigma,-s}, 1 \leq j \leq 2^{s}$, so that their union is $q$ and associate with each $Q$ assigned to $q$ one $q_{j}$ such that $Q$ intersects $q_{j}$ (hence $Q \subset q_{j}^{*}$ ). Write

$$
A_{q_{j}}=\sum_{Q \text { associated to } q_{j}} \lambda_{Q} a_{Q}, \quad \lambda_{q_{j}}=\sum_{Q \text { associated to } q_{j}} \lambda_{Q} .
$$


We then have $A_{q}=\sum_{j=1}^{2^{s}} A_{q_{j}}, \lambda_{q}=\sum_{j=1}^{2^{s}} \lambda_{q_{j}}$. We now write the left-hand side of (7) as

$$
\begin{aligned}
& \left\|\sum_{\sigma<-s} \sum_{q \in R_{\sigma, 0}} A_{q} * K_{0}\right\|_{L^{2}}^{2} \\
& \quad=2 \sum_{\sigma^{\prime}<-s} \sum_{\sigma \leq \sigma^{\prime}} \sum_{q^{\prime} \in R_{\sigma^{\prime}, 0}} \sum_{q \in R_{\sigma, 0}} \int\left(A_{q} * K_{0}\right)\left(A_{q^{\prime}} * K_{0}\right) d z d \bar{z} d t \\
& \quad \leq \mathrm{I}+\mathrm{II},
\end{aligned}
$$

where

$$
\begin{aligned}
& \mathrm{I}=2 \sum_{\sigma^{\prime}<-s} \sum_{\sigma \leq \sigma^{\prime}} \sum_{q^{\prime} \in R_{\sigma^{\prime}, 0}} \sum_{\substack{q \in R_{\sigma, 0} \\
q \cap\left(q^{\prime}\right)^{*} \neq \varnothing}}\left|\int\left(A_{q} * K_{0}\right)\left(A_{q^{\prime}} * K_{0}\right) d z d \bar{z} d t\right|, \\
& \mathrm{II}=2 \sum_{\sigma^{\prime}<-s} \sum_{\sigma \leq \sigma^{\prime}} \sum_{q^{\prime} \in R_{\sigma^{\prime}, 0}} \sum_{\substack{q \in R_{\sigma, 0} \\
q \cap\left(q^{\prime}\right)^{*}=\varnothing}}\left|\int\left(A_{q} * K_{0}\right)\left(A_{q^{\prime}} * K_{0}\right) d z d \bar{z} d t\right| .
\end{aligned}
$$

To treat term I, let us fix $\sigma^{\prime}<-s, \sigma \leq \sigma^{\prime}, q^{\prime} \in R_{\sigma^{\prime}, 0}$. Then

$$
\begin{aligned}
& \sum_{\substack{q \in R_{\sigma, 0} \\
q \cap\left(q^{\prime}\right)^{*} \neq \varnothing}}\left|\int\left(A_{q} * K_{0}\right)\left(A_{q^{\prime}} * K_{0}\right) d z d \bar{z} d t\right| \\
& \quad \leq \sum_{\substack{q \in R_{\sigma, 0} \\
q \cap\left(q^{\prime}\right)^{*} \neq \varnothing}}\left\|A_{q^{\prime}} * K_{0}\right\|_{L^{1}}\left\|A_{q} * K_{0}\right\|_{L^{\infty}} \quad \text { (by Lemma 3) } \\
& \leq \sum_{\substack{q \in R_{\sigma, 0} \\
q \cap\left(q^{\prime}\right)^{*} \neq \varnothing}} C \lambda_{q^{\prime}} 2^{-\sigma} \lambda_{q} .
\end{aligned}
$$

To get a bound for (12) write $\left(q^{\prime}\right)^{*} \cap$ (vertical Euclidean cylinder over $q^{\prime}$ ) as a union of $C 2^{s}, q_{j}^{\prime} \in R_{\sigma,-s}$. We then have that $\bigcup_{j}\left(q_{j}^{\prime}\right)^{*} \supseteq\left(q^{\prime}\right)^{*}$ and therefore

$$
\sum_{\substack{q \in R_{\sigma, 0} \\ q \cap\left(q^{\prime}\right)^{* \neq \neq} \neq \varnothing}} \lambda_{q} \leq \sum_{j=1}^{C 2^{s}} \sum_{\substack{q \in R_{\sigma, 0} \\ q \cap\left(q_{j}^{\prime}\right)^{* \neq \neq} \neq \varnothing}} \lambda_{q} \leq \sum_{j=1}^{C 2^{s}} \sum_{\substack{q \in R_{\sigma, 0} \\ q \subseteq\left(q_{j}^{\prime}\right)^{* *}}} \lambda_{q} \leq \sum_{j=1}^{C 2^{s}} \sum_{\substack{Q \subseteq\left(q_{j}^{\prime}\right)^{* * *} \\ \kappa(Q)=-s}} \lambda_{Q} \cdot
$$

An application of (2.4) for each $q_{j}^{\prime}$ gives that the above is bounded by

$$
C 2^{s} 2^{n+1} \alpha 2^{\sigma-(2 n+1) s} \leq C \alpha 2^{\sigma-2 n s} .
$$

Therefore (12) is bounded above by $C \lambda_{q^{\prime}} 2^{-\sigma} C \alpha 2^{\sigma-2 n s} \leq C \alpha \lambda_{q^{\prime}} 2^{-s}$.

Summing over all $q^{\prime} \in R_{\sigma^{\prime}, 0}, \sigma \leq \sigma^{\prime}$, and $\sigma^{\prime}<-s$ we get the desired conclusion for I.

In the sequel we will need the following simple lemma whose proof we omit. 
Lemma 5. On the Heisenberg group, let a have support contained in the set $A$ and integral 0 and let $h$ be $C^{1}$. Then

$$
|(a * h)(u)| \leq\|a\|_{L^{1}}\|\nabla h\|_{L^{\infty}\left(A^{-1} \cdot u\right)} \text { Euclidean diameter of }\left(A^{-1} \cdot u\right) .
$$

To treat term II, fix as before $\sigma^{\prime}<-s, \sigma \leq \sigma^{\prime}, q^{\prime} \in R_{\sigma^{\prime}, 0}$. Note that for $q \in R_{\sigma, 0}$ and all $u \in \mathbb{H}^{n}$, the Euclidean diameter of $q^{-1} u$ is at most $C 2^{-s}$. We have

$$
\begin{aligned}
& \sum_{\substack{q \in R_{\sigma, 0} \\
q \cap\left(q^{\prime}\right)^{*}=\varnothing}}\left|\int\left(A_{q} * K_{0}\right)\left(A_{q^{\prime}} * K_{0}\right) d z d \bar{z} d t\right| \\
& \leq \sum_{\substack{r \in R_{\sigma,-s} \\
r \cap\left(q^{\prime}\right)^{*}=\varnothing}}\left|\int\left(A_{r} * K_{0}\right)\left(A_{q^{\prime}} * K_{0}\right) d z d \bar{z} d t\right| \\
& \leq \sum_{\substack{q \in R_{\sigma}=s \\
q \cap\left(q^{\prime}\right)^{*}=\varnothing}}\left|\int A_{q}\left(A_{q^{\prime}} * K_{0} * \widetilde{K}_{0}\right) d z d \bar{z} d t\right| \\
& \leq \sum_{\substack{q \in R_{\sigma^{\prime}}=s \\
q \cap\left(q^{\prime}\right)^{*}=\varnothing}} \lambda_{q^{\prime}}\left\|A_{q} * K_{0} * \widetilde{K}_{0}\right\|_{L^{\infty}\left(q^{\prime}\right)} \quad \text { (by Lemma 5) } \\
& \leq C 2^{-s} \lambda_{q^{\prime}} \sum_{\substack{q \in R_{\sigma^{\prime}-s} \\
q \cap\left(q^{\prime}\right)^{*}=\varnothing}} \lambda_{q}\left\|\nabla\left(K_{0} * \widetilde{K}_{0}\right)\right\|_{L^{\infty}\left(q^{-1} \cdot q^{\prime}\right)} .
\end{aligned}
$$

To get an estimate for (13) consider all expansions of $q^{\prime}$ by a factor of $2^{m}$, $m \geq 0$. By this we mean all sets $q_{m}^{\prime}$ in $\widetilde{R}_{m+\sigma^{\prime}, 0}$ with the same center as $q^{\prime}$ and tilted as $q^{\prime}$. We first fix an $m \geq 0$ and get an estimate for

$$
\sum_{\substack{q \in R_{o,-s} \\ q \cap q_{m+1}^{\prime} \neq \varnothing}} \lambda_{q} .
$$

Note that since $K_{0} * \widetilde{K}_{0}$ has compact support only those $m$ for which $m+\sigma^{\prime} \leq C$ are of interest to us. Consider two cases:

Case 1. $m+\sigma^{\prime}<0$. Write $q_{m+1}^{\prime}$ as the union of $2^{-\left(m+\sigma^{\prime}\right)}$ rectangles $q_{m+1, k}^{\prime} \in$ $R_{m+\sigma^{\prime}, m+\sigma^{\prime}}, 1 \leq k \leq 2^{m+\sigma^{\prime}}$. Then

$$
\begin{aligned}
\sum_{\substack{q \in R_{\sigma,-s} \\
q \cap q_{m+1}^{\prime} \neq \varnothing}} \lambda_{q} \leq \sum_{k=1}^{2^{-\left(m+\sigma^{\prime}\right)}} \sum_{\substack{q \in R_{\sigma,-s} \\
q \cap q_{m+1, k}^{\prime} \neq \varnothing}} \lambda_{q} \leq \sum_{k=1}^{2^{-\left(m+\sigma^{\prime}\right)}} \sum_{Q \subset\left(q_{m+1, k}^{\prime}\right)} \lambda_{q} \\
\leq 2^{-\left(m+\sigma^{\prime}\right)} C \alpha 2^{m+\sigma^{\prime}+(2 n+1)\left(m+\sigma^{\prime}\right)}=C \alpha 2^{(2 n+1)\left(m+\sigma^{\prime}\right)},
\end{aligned}
$$

where in the last inequality we used (2.4). 
Case 2. $0 \leq m+\sigma^{\prime} \leq C$. All $q \in R_{\sigma,-s}$ which intersect $q_{m+1}^{\prime}$ intersect at most $C$ rectangles $R_{k}$ in $R_{0,0}$. Then

$$
\sum_{\substack{q \in R_{\sigma,-s} \\ q \cap q_{m+1}^{\prime} \neq \varnothing}} \lambda_{q} \leq \sum_{k=1}^{C} \sum_{\substack{q \in R_{\sigma,-s} \\ q \cap R_{k} \neq \varnothing}} \lambda_{q} \leq \sum_{k=1}^{C} \sum_{\substack{Q \subseteq R_{k}^{* *} \\ R_{k} \in R_{0,0}}} \lambda_{Q} \leq \dot{C} \alpha .
$$

We finally get an estimate for (13):

$$
\begin{aligned}
\text { (13) } & \leq C 2^{-s} \lambda_{q^{\prime}} \sum_{m=0}^{C-\sigma^{\prime}} \sum_{\substack{q \in R_{\sigma,-s} \\
q \cap\left(q_{m+1}^{\prime}-q_{m}^{\prime}\right) \neq \varnothing}} \lambda_{q}\left\|\nabla\left(K_{0} * \widetilde{K}_{0}\right)\right\|_{L^{\infty}\left(q^{-1} \cdot q^{\prime}\right)} \\
& \leq C 2^{-2} \lambda_{q^{\prime}} \sum_{m=0}^{C-\sigma^{\prime}} \sum_{\substack{q \in R_{\sigma,-s} \\
q \cap q_{m+1}^{\prime} \neq \varnothing}} \lambda_{q} 2^{-2\left(m+\sigma^{\prime}\right)} \\
& \leq C 2^{-s} \lambda_{q^{\prime}}\left[\sum_{m=0}^{-\sigma^{\prime}} 2^{-2\left(m+\sigma^{\prime}\right)} C \alpha 2^{(2 n+1)\left(m+\sigma^{\prime}\right)}+\sum_{m=-\sigma^{\prime}+1}^{C-\sigma^{\prime}} 2^{-2\left(m+\sigma^{\prime}\right)} C \alpha\right] \\
& \leq C \alpha 2^{-s} \lambda_{q^{\prime}} .
\end{aligned}
$$

Summing over all $q^{\prime} \in R_{\sigma^{\prime}, 0}$, all $\sigma \leq \sigma^{\prime}$, and all $\sigma^{\prime}<-s$ we get the desired conclusion for term II. This concludes the proof of (7).

\section{THE OFF-DIAGONAL TERMS}

This section is devoted to the proof of (8). We begin with a lemma.

Lemma 6. For $k \leq-3, K_{k} * \widetilde{K}_{0}$ is supported in the set $\{(z, t):|z| \leq C,|t| \leq$ $\left.C 2^{k}\right\}$ and satisfies

$$
\begin{gathered}
\left\|K_{k} * \widetilde{K}_{0}\right\|_{L^{\infty}} \leq C 2^{-k}, \\
\left\|\nabla\left(K_{k} * \widetilde{K}_{0}\right)\right\|_{L^{\infty}} \leq C 2^{-2 k}, \\
\left\|\nabla^{2}\left(K_{k} * \widetilde{K}_{0}\right)\right\|_{L^{\infty}} \leq C 2^{-3 k} .
\end{gathered}
$$

Proof. The same reasoning as in Lemma 4 shows that, when $l=n$,

$$
\left(K_{k} * \widetilde{K}_{0}\right)(z, t)=\frac{1}{4}\left|z_{n}\right|^{-2} \int_{\mathbb{C}^{n-1}} \int_{\mathbb{R}} L_{k}(z+\tilde{\zeta}) L_{0}(\tilde{\zeta}) d s d \zeta^{\prime} d \bar{\zeta}^{\prime},
$$

where $\zeta^{\prime}=\left(\zeta_{1}, \ldots, \zeta_{n-1}\right) \in \mathbb{C}^{n-1}$ and

$$
\tilde{\zeta}=\left(\zeta_{1}, \ldots, \zeta_{n-1}, \frac{s-i t+2 \bar{z}^{\prime} \cdot \zeta^{\prime}}{-2 \bar{z}_{n}}\right) \in \mathbb{C}^{n} .
$$

Since $L_{k}(z)$ is supported in $|z| \sim 2^{k}$ we get that

$$
\left|z^{\prime}+\zeta^{\prime}\right|+\left|z_{n}+\frac{s-i t+2 \bar{z}^{\prime} \cdot \zeta^{\prime}}{-2 \bar{z}_{n}}\right| \leq C 2^{k}
$$


thus $\zeta^{\prime}$ integrates over a Euclidean ball about $-z^{\prime}$ of radius $C 2^{k}$. The above inequality gives

$$
\begin{gathered}
\left.|s-2| z_{n}\right|^{2}+2 \operatorname{Re} \bar{z}^{\prime} \cdot \zeta^{\prime}\left|\leq C 2^{k}\right| z_{n} \mid, \\
\left|t-2 \operatorname{Im} \bar{z}^{\prime} \cdot \zeta^{\prime}\right| \leq C 2^{k}\left|z_{n}\right| .
\end{gathered}
$$

We certainly have that $\left|z_{n}\right| \leq C$ on the support of $\left(K_{k} * \widetilde{K}_{0}\right)(z, t)$. It follows that for any fixed $\zeta^{\prime}, s$ integrates over a set of measure $\leq C 2^{k}$. Also

$$
\begin{aligned}
|t| & \leq C 2^{k}\left|z_{n}\right|+\left|2 \operatorname{Im} \bar{z}^{\prime} \cdot \zeta^{\prime}\right| \\
& \leq C 2^{k}+2\left|\operatorname{Im}\left(\bar{z}+\bar{\zeta}^{\prime}\right) \cdot \zeta^{\prime}\right| \\
& \leq C 2^{k}+2\left|z^{\prime}+\zeta^{\prime}\right|\left|\zeta^{\prime}\right| \leq C 2^{k} .
\end{aligned}
$$

This proves the assertion about the support of $K_{k} * \widetilde{K}_{0}$.

We now prove the size estimates for $K_{k} * \widetilde{K}_{0}$. It follows from the definition of $\tilde{\zeta}$ that the plane $z_{n}=0$ does not intersect the support of $K_{k} * \widetilde{K}_{0}$. Since the latter set is compact, there exists a constant $C_{0}$ such that $\left|z_{n}\right| \geq C_{0}$ when $(z, t)$ lies in the support of $K_{k} * \widetilde{K}_{0}$. We use $\left\|L_{k}\right\|_{L^{\infty}} \leq C\left(2^{k}\right)^{-2 n}$ to estimate $\left\|K_{k} * \widetilde{K}_{0}\right\|_{L^{\infty}}$ by

$$
\begin{aligned}
& \frac{1}{4} C_{0}^{-2} \int_{\left|\zeta^{\prime}+z^{\prime}\right| \leq C 2^{k}} \int_{\left|s-a_{\zeta^{\prime}}\right| \leq C 2^{k}} \int_{\left|s-a_{\zeta^{\prime}}\right| \leq C 2^{k}}\left\|L_{k}\right\|_{L^{\infty}}\left\|L_{0}\right\|_{L^{\infty}} d s d \zeta^{\prime} d \bar{\zeta}^{\prime} \\
& \leq C\left(2^{k}\right)^{2(n-1)} 2^{k}\left(2^{k}\right)^{-2 n}=C 2^{-k},
\end{aligned}
$$

where in the above estimate we set $a_{\zeta^{\prime}}=2\left|z_{n}\right|^{2}-2 \operatorname{Re} \bar{z}^{\prime} \cdot \zeta^{\prime}$. The estimates for the derivatives of $K_{k} * \widetilde{K}_{0}$ are similar.

For each $k \leq-3$, let us call $S_{k}=\left\{(z, t):|z| \leq C,|t| \leq C 2^{k}\right\}$ so that support $\left(K_{k} * \widetilde{K}_{0}\right) \subseteq S_{k}$. Note that for each $u \in \mathbb{H}^{n}$ we have $S_{k}^{-1} u=S_{k} u$.

We will need the following two lemmas.

Lemma 7. Fix any $Q \in \mathscr{C}$ with $\sigma(Q)=\sigma$ and any $k \leq-3$. If $m=\max (\sigma, k)$ we have

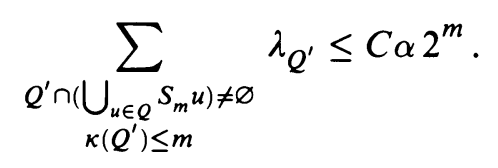

Lemma 8. For any $Q \in \mathscr{C}$ with $\sigma(Q)=\sigma$, any $\kappa \leq \sigma$, and any $(w, s) \in \mathbb{H}^{n}$,

$$
\int_{Q} \chi_{S_{k}(z, t)}(w, s) d z d \bar{z} d t \leq C 2^{k+(2 n+1) \sigma} .
$$

Proof. For any $u \in Q, S_{m} u$ is a tilted rectangle in $\mathbb{R}^{2 n+1}$ of dimensions

$$
(\underbrace{C, \ldots, C}_{2 n \text { times }}, C 2^{m}) \text {. }
$$


It follows that $\bigcup_{u \in Q} S_{m} u$ is contained in a tilted rectangle in $\mathbb{R}^{2 n+1}$ of dimensions

$$
(\underbrace{C, \ldots, C}_{2 n \text { times }}, C 2^{m}) \text {. }
$$

Cover $\bigcup_{n \in Q} S_{m} u$ by the union of $C 2^{-(2 n+1) m}$ elements of $R_{m, m}$. For each element of $R_{m, m}$ apply (2.4). The desired conclusion follows.

To prove (8.1) note that the set of all $(z, t) \in Q$ for which the fixed $(w, s)$ lies in $S_{k}(z, t)$ is contained in a rectangle $R$ of dimensions

$$
(\underbrace{C, \ldots, C}_{2 n \text { times }}, C 2^{k})
$$

centered at the center of $Q$ and with maximum tilt from its center $\sim C d$, where $d$ denotes the Euclidean distance from the center of $Q$ to the origin. $Q$ has dimensions

$$
(\underbrace{2^{\sigma}, \ldots, 2^{\sigma}}_{2 n \text { times }}, 2^{2 \sigma})
$$

and has maximum tilt from its center $\sim C 2^{\sigma} d(\sigma<0)$. It follows that $Q$ and $R$ intersect almost vertically and with respect to the coordinate system induced by $R, Q$ should be thought of as having dimensions $\left(2^{\sigma}, \ldots, 2^{2 \sigma}, \ldots, 2^{\sigma}\right)$. Therefore $Q \cap R$ has measure at most

$$
C \underbrace{2^{\sigma}, \ldots, 2^{\sigma}}_{2 n-1 \text { times }}, 2^{2 \sigma} 2^{\min (k, \sigma)}=C 2^{k+(2 n+1) \sigma} .
$$

We can now prove (8). Write the left-hand side of (8) as:

$$
\begin{aligned}
& \sum_{k \leq-3} \int\left(B_{-s}\right)\left(B_{k-s} * K_{k} * \widetilde{K}_{0}\right) d z d \bar{z} d t \\
& \quad \leq \sum_{\substack { \sigma<-s \\
\begin{subarray}{c}{\kappa(Q)=-s \\
\sigma(Q)=\sigma{ \sigma < - s \\
\begin{subarray} { c } { \kappa ( Q ) = - s \\
\sigma ( Q ) = \sigma } }\end{subarray}} \lambda_{Q} \sum_{k \leq-3}\left|\int\left(A_{Q}\right)\left(B_{k-s} * K_{k} * \widetilde{K}_{0}\right) d z d \bar{z} d t\right| \\
& \quad \leq \mathrm{I}+\mathrm{II},
\end{aligned}
$$

where

$$
\begin{aligned}
& \mathrm{I}=\sum_{\sigma<-s} \sum_{\substack{\boldsymbol{\alpha}(Q)=-s \\
\sigma(Q)=\sigma}} \sum_{k \leq \sigma} \lambda_{Q} \int\left|A_{Q}\right|\left|B_{k-s} * K_{k} * \widetilde{K}_{0}\right| d z d \bar{z} d t, \\
& \mathrm{II}=\sum_{\sigma<-s} \sum_{\substack{\alpha(Q)=-s \\
\sigma(Q)=\sigma}} \sum_{k \leq \sigma} \lambda_{Q}\left|\int\left(A_{Q}\right)\left(B_{k-s} * K_{k} * \widetilde{K}_{0}\right) d z d \bar{z} d t\right| .
\end{aligned}
$$

We begin with term I. Fix $\sigma<-s$ and $Q \in \mathscr{C}$ with $\kappa(Q)=-s$ and $\sigma(Q)=\sigma$. 
By Lemma 5 and the definition of an atom we get

$$
\begin{aligned}
& \sum_{k \leq \sigma} \lambda_{Q} \int\left|a_{Q}\right|\left|B_{k-s} * K_{k} * \widetilde{K}_{0}\right| d z d \bar{z} d t \\
& \quad \leq C \lambda_{Q}|Q|^{-1} \sum_{k \leq \sigma} \int_{Q}\left\{\sum_{\substack{\kappa\left(Q^{\prime}\right)=k-s \\
Q^{\prime} \cap S_{k}^{-1}(z, t) \neq \varnothing}} \lambda_{Q^{\prime}} 2^{\sigma\left(Q^{\prime}\right)}\left\|\nabla\left(K_{k} * \widetilde{K}_{0}\right)\right\|_{L^{\infty}}\right\} d z d \bar{z} d t
\end{aligned}
$$

(by (2.3) and (6.2))

$$
\begin{aligned}
& \leq C \lambda_{Q}|Q|^{-1} \sum_{k \leq \sigma} 2^{k-s} 2^{-2 k} \int_{Q} \sum_{\substack{\kappa\left(Q^{\prime}\right)=k-s \\
Q^{\prime} \cap S_{k}(z, t) \neq \varnothing}} \lambda_{Q^{\prime}} d z d \bar{z} d t \\
& \leq C \lambda_{Q}|Q|^{-1} \sum_{k \leq \sigma} 2^{-k-s} \int_{Q}\left[\int \sum_{\substack{Q^{\prime} \cap S_{k}(z, t) \neq \varnothing \\
\kappa\left(Q^{\prime}\right)=k-s}} \lambda_{Q^{\prime}\left|Q^{\prime}\right|^{-1} \chi_{Q^{\prime}}(w, s)}\right] \\
& \left.\cdot \chi_{S_{k}(z, t)}(w, s) d w d \bar{w} d s\right] d z d \bar{z} d t
\end{aligned}
$$

(by Fubini)

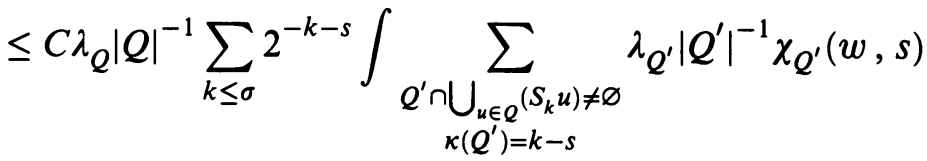

$$
\begin{aligned}
& \cdot\left[\int_{Q} \chi_{S_{k}(z, t)}(w, s) d z d \bar{z} d t\right] d w d \bar{w} d s
\end{aligned}
$$

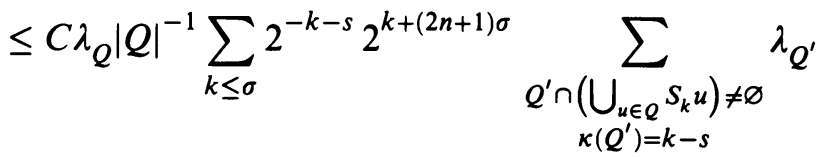

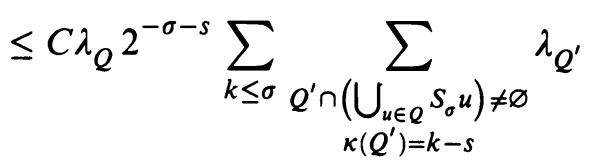

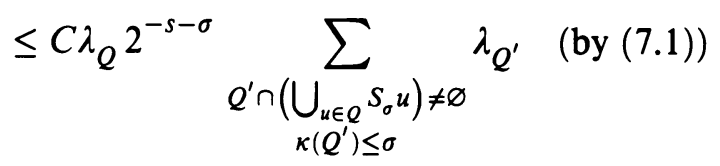

$\leq C \alpha 2^{-s} \lambda_{Q}$ 
Summing over all $Q \in \mathscr{C}$ with $\sigma(Q)=\sigma, \kappa(Q)=-s$, and all $\sigma<-s$ we get the required conclusion for term $I$.

To treat term II, fix $\sigma<-s$ and $Q \in \mathscr{C}$ with $\sigma(Q)=\sigma$ and $\kappa(Q)=-s$. Two applications of Lemma 5 give the first two inequalities below.

$$
\begin{aligned}
& \sum_{k>\sigma} \lambda_{Q}\left|\int A_{Q}\left(B_{k-s} * K_{k} * \widetilde{K}_{0}\right) d z d \bar{z} d t\right| \\
& \leq C \sum_{k>\sigma} \lambda_{Q} 2^{\sigma}\left\|B_{k-s} * \nabla\left(K_{k} * \widetilde{K}_{0}\right)\right\|_{L^{\infty}(Q)} \\
& \leq C \sum_{k>\sigma} \lambda_{Q^{2}} 2^{\sigma} \sup _{u \in Q} \sum_{\substack{Q^{\prime} \cap S_{k}^{-1} u \neq \varnothing \\
\kappa\left(Q^{\prime}\right)=k-s}} \lambda_{Q^{\prime}} 2^{\sigma\left(Q^{\prime}\right)}\left\|\nabla^{2}\left(K_{k} * k_{0}\right)\right\|_{L^{\infty}} \\
& \text { (by (2.3) and (6.3)) } \\
& \leq C \sum_{k>\sigma} \lambda_{Q} 2^{\sigma} 2^{k-s} 2^{-3 k} \sup _{u \in Q} \sum_{\substack{Q^{\prime} \cap\left(\bigcup_{u \in \in} S_{k} u\right) \neq \varnothing \\
\kappa\left(Q^{\prime}\right)=k-s}} \lambda_{Q^{\prime}} \\
& \leq C \lambda_{Q^{2}} 2^{-s} \sum_{k>\sigma} 2^{\sigma-2 k} \sum_{Q^{\prime} \cap\left(\bigcup_{\substack{u \in Q \\
\kappa\left(Q^{\prime}\right) \leq k}} S_{k} u\right) \neq \varnothing} \lambda_{Q^{\prime}} \\
& \leq C \lambda_{Q} 2^{-s} \sum_{k>\sigma} 2^{\sigma-2 k}\left(C \alpha 2^{k}\right) \leq C \alpha \lambda_{Q} 2^{-s} .
\end{aligned}
$$

The penultimate inequality follows by another application of (7.1). Summing over all $Q \in \mathscr{C}$ with $\sigma(Q)=\sigma, \kappa(Q)=-s$, and all $\sigma<-s$ we get the required conclusion for term II. (8) is now proved. This concludes the proof of (1) and hence of our theorem.

\section{CONCLUDING REMARKS}

The proof follows the method initiated in $[C]$ in the treatment of the maximal function along the parabola $\left(t, t^{2}\right)$. An application of this method gives Theorem 2 in [G]. The same method might prove that the analytic family $A^{\gamma}$ considered by Geller and Stein in [GS2] maps $H^{1}$ to $L^{1, \infty}$ when $\operatorname{Re} \gamma=0$. This result, together with the sharp $L^{2}$ estimates in [GS2], would give a positive endpoint result for the analytic family $A^{\gamma}$. It still remains an open question whether the operator $A$ is of weak type $(1,1)$. The answer to this problem is probably the same as for the Hilbert transform along the parabola $\left(t, t^{2}\right)$ in $\mathbb{R}^{2}$.

I would like to thank my supervisor Mike Christ, for introducing me to this problem and for giving me numerous suggestions throughout this work. 


\section{REFERENCES}

[CC] S. Chaneilo and M. Christ, Weak type $(1,1)$ bounds for oscillatory integrals, Duke Math J. 55 (1987), 141-155.

[C] M. Christ, Weak type $(1,1)$ bounds for rough operators, Ann. of Math. (2) 128 (1988), 19-42.

[CG] M. Christ and D. Geller, Singular integral characterizations of Hardy spaces on homogeneous groups, Duke Math. J. 51 (1984), 547-598.

[CR] M. Christ and J. L. Rubio de Francia, Weak type $(1,1)$ bounds for rough operators. II, Invent. Math. 93 (1988), 225-237.

[CW] R. Coifman and Guido Weiss, Analyse harmonique non-commutative sur certains espaces homogenes, Springer-Verlag, Berlin-Heidelberg-New York, 1971.

[FS1] G. Folland and E. Stein, Hardy spaces on homogeneous groups, Princeton Univ. Press, Princeton, N.J., 1982.

[FS2] _ Estimates for the $\bar{\partial}_{b}$ complex and analysis on the Heisenberg group, Comm. Pure Appl. Math. 27 (1974), 429-522.

[GS1] D. Geller and E. Stein, Singular convolution operators on the Heisenberg group, Bull. Amer. Math. Soc. (N.S.) 6 (1982), 99-103.

[GS2] _ Estimates for singular convolution operators on the Heisenberg group, Math. Ann. 267 (1984), 1-15.

[G] L. Grafakos, Endpoint bounds for an analytic family of Hilbert transforms, Duke Math. J. (to appear).

[S] E. Stein, Singular integrals and differentiability properties of functions, Princeton Univ. Press, Princeton, N.J., 1970.

Department of Mathematics, Box 2155 Yale Station, Yale University, New Haven, CONNECTICUT 06520 